\title{
ESTUDIOS INMUNOLOGICOS EN HAMSTERS (CRICETUS AURATUS) INFECTADOS CON SCHISTOSOMA MANSQNI (1)
}

\section{R E S U M E N}

Los resultados de este trabajo muestran que el hamster (Cricetus auratus) puede ser utilizado como un modelo experimental para estudios inmunológicos en la infección por Schistosoma mansoni. Los datos obtenidos, relativos a inmu. nidad concomitante, producción de anticuerpo letal e inmunosupresión se asemejan a los conseguidos en otros modelos experimentales ya establecidos. Estas observaciones indican que el hamster, además de ser un hospedero satisfactorio para el mantenimiento del parásito en el laboratorio, puede ser considerado como un modelo experimental alterno cuyo crecimiento y mantenimiento son relativamente simples y además es un animal de fácil manejo.

UNITERMOS: Esquistosomfase mansonica experimental - Hamster (Cricetus auratus) - Inmunologia

\section{N T RODU C I I N}

E1 desarrollo de un modelo experimental para el estudio de los differentes aspectos inmunológicos de las infecciones por Schistosoma mansoni constituye una preocupación de los in. vestigadores interesados en el control de la esquistosomiasis.

De los animales de laboratorio disponibles, únicamente los ratones parecen ser los hospederos adecuados para el estudio, en gran escala, de los diversos fenómenos inmunológicos presentes en la esquistosomiasis. Cobayos pre. sentan respuesta inmunológica semejante al de los ratones pero no se consigue detectar huevos en las heces de estos animales, de ma. nera que el ciclo evolutivo del Schistosoma no puede ser completado en este hospedero 12,13. Los monos, otros posibles hospederos, son difíciles de adquirir y mantener, mientras que las ratas y los conejos no son buenos hospede- ros y la respuesta inmunológica es muy diferente a la que ocurre en el hombre 24.

Por este motivo, nos propusimos analisar algunos aspectos inmunológicos en hamsters (Cricetus auratus) infectados con $\mathbf{S}$. mansoni, con el fin de establecer si tales animales, que generalmente se han utilizado sólo para mantener la cepa del parásito en el laboratorio, pueden constituir un modelo alterno experimental para la esquistosomiasis mansoni.

Pensando en lo anterior, los objetivos del trabajo fueron observar en el hamster el desarrollo de inmunidad adquirida en el curso de la infección primaria por S. mansoni; indagar sobre la presencia de anticuerpo letal ${ }^{3}$ y su relación con el desarrollo de la inmunidad protectora. También fueron contemplados en este estudio fenómenos de inmunosupresión.

(1) Este trabajo fue financiado, on parte, por el "Conselho Nacional de Desenvolvimento Científico e Tecnológico do Brasil" e FINEP (Financiadora de Estudos a Projetos).

(2) Departamento de Parasitología, Universidad de Costa Rica, San José, Costa Rica

(3) Departamento de Parasitologia, Instituto de Ciências Blológicas, UFMG, Belo Forizonte, Brasil

(4) Departamento de Bloquimica-Imunologia, Instituto de Ciências Biológicas, UFMG, Belo Horizonte, Brasil 
MONGE, E.; COELHO, P. M. Z. \& TAVARES, C. A. P. - Estudios inmunologicos en hamsters (Cricetus auratus) infectados con Schistosoma mansoni. Rev. Inst. Med. trop. Sāo Paulo, 28:239.245, 1986.

\section{MATERIA】 Y METODOS}

Se utilizaron 200 hamsters machos, heterogenéticos, de un mes y medio a dos meses y medio de edad cuyo peso varió entre 70 y 90 gramos, procedentes del bioterio del Departa. mento de Parasitologia de la Universidad Federal de Minas Gerais (ICB-UFMG) ý mantenidos con alimento y agua ad libitum.

Las cercarias de S. mansoni (cepa L.E., Belo Horizonte, Brasil), obtenidas de Biomphala ria glabrata creados en el laboratorio, fueron concentradas y a partir de ellas se obtuvieron esquistosómulos in vitro según la técnica de RAMALHO-PINTO \& col. 15.

Se infectaron 150 hamsters con 30 cercarias por via transcutánea de acuerdo con la técnica de SMITHERS \& TERRY ${ }^{19}$. Posteriormente cuatro grupos de 12 animales cada uno se reinfectó con 150 cercarias a los 40,60 y 90 días y con 250 a los 120 días. Para cada grupo se empleó un grupo testigo de 12 hamsters que sólo fueron expuestos a las dosis de 150 o 250 cercarias según el caso.

Catorce días después de la reinfección los animales fueron sacrificados por fractura cervical, recuperándose los vermes por perfusión del sistema porta empleando un aparato para perfusión (Brewer Automatic Pipetting Machine, Mar. U.S.A.). Del material, recogido en un beaker, se obtuvieron los vermes que fueron contados en una placa de Petri bajo el estereos. copio. La diferenciación de las dos infecciones se basó en el tamaño y la morfologia de los vermes y de acuerdo con el número de los in. maduros recuperados, se evalúo la resistencia de los hamsters a la reinfección, que se expresó en porcentaje de reducción 16 .

Con la finalidad de estimar la acción del anticuerpo letal presente en el suero de hamsters infectados con S. mansoni, sobre esquistosómulos de 90 minutos, los animales reinfectar dos a los 40,60, 90 y 120 días después de la infección fueron sangrados al igual que hamsters no infectados los cuales se usaron como testigos. La sangre fue obtenida del plexo retrorbital con ayuda de una pipeta Pasteur y el suero extraído por centrifugación y mantenido a $-20^{\circ} \mathrm{C}$ fue usado en la determinación de anticuerpo letal, según la técnica descrita por TAVARES \& col. ${ }^{22}$. También se determinó el ti- tulo de anticuerpo letal mezclando los sueros obtenidos a $\operatorname{los} 40,60,90$, y 120 días después de infección. La acción citotóxica fue estimada por la determinación del índice de mortalidad de los esquistosómulos por diferencia en. tre los muertos por el suero inmune más complemento y los muertos sólo por la acción del complemento.

La evaluación de la respuesta celular (hipersensibilidad retardada) fue realizada a los 45,60 y 90 días después de la infección inicial tanto en animales infectados o no con $\mathbf{S}$. mansoni, de acuerdo con la técnica de MILLER \& col. ${ }^{8}$ que usa glóbulos rojos de carnero como indicador de hipersensibilidad retardada. El gra. do de hichazón d elas patas fue determinado por medio de un micrómetro ajustado a lecturas de centésimo de milimetro (L.S. Starret, Co., Gran Bretaña, 10 que permitió establecer las diferencias del edema de las patas posteriores.

Para evaluar el grado de significancia estadística, los resultados fueron sometidos a la prueba de $t$ de Student, a análisis de variancia $y$ de correlación linear.

\section{RESULTADOS}

El porcentaje de adultos recuperados en relación al número total de cercarias utilizadas en la infección fue establecido con base en el total y el promedio de vermes de cada sexo, recuperados de los hamsters infectados (Cuadro I).

El Cuadro II contiene los promedios y las respectivas desviaciones estándares del número de vermes inmaduros de $\mathbf{S}$. mansoni, recuperados después de la reinfección de los di. ferentes grupos de animales. Como se puede observar hubo una diferencia significativa a par. tir de los 60 días después de la infección inicial cuando comparados con los testigos.

Los porcentajes de reducción de la carga parasitaria están representados en la figura 1 Por análisis de variancia se verificó que la re ducción fue significativa a un nivel de $1 \%$ en tre 40 y 60 días y de $0,1 \%$ entre 40 y 90 y 40 y 120 días pero no lo fue entre 60 y 90 y 60 y 120 días indicando que después de los 60 días la re ducción de la carga parasitaria fue práctica. mente la misma. 
MONGE, E.; COELHO, P. M. Z. \& TAVARES, C. A. P. - Estudios inmunologicos en hamsters (Cricetus auratus) infectados con Schistosoma mansoni. Rev. Inst. Med. trop. Săo Paulo, 28:239-245, 1986.

C U A D R O I

Numero y porcentaje de vermes aduitos de Schistosoma mansoni recuperados de hamsters después de la infección primaria

\begin{tabular}{cccccc}
\hline $\begin{array}{l}\text { Dfas después } \\
\text { 1a. infección }\end{array}$ & $\begin{array}{c}\text { No. de } \\
\text { hamsters }\end{array}$ & \multicolumn{2}{c}{ Parásitos recuperados } & Promedio \pm D.E. & Recuperación (\%) \\
\hline 40 & machos & hembras & \\
60 & 12 & 85 & 52 & $11,4 \pm 3,6$ & $38,1 \pm 3,6$ \\
90 & 11 & 74 & 36 & $10,0 \pm 4,1$ & $33,3 \pm 4,0$ \\
120 & 10 & 66 & 43 & $10,9 \pm 3,9$ & $36,3 \pm 4,0$ \\
Total & 12 & 100 & 62 & $12,7 \pm 3,9$ & $42,2 \pm 3,9$ \\
& 45 & 325 & 183 & $11,3 \pm 3,9$ & $\overline{\mathbf{X}} 37,5 \pm 3,7\left(^{*}\right)$ \\
\hline
\end{tabular}

Los hamsters fueron infectados con cerca de 30 cercarias de Schistosoma mansoni.

(*) Representa el promedio y la desviación estandar de Ios porcentajes de recuperación.

C U A D R O II

Verificación de la inmunidad concomitante en hamsters infectados inicialmente con cerca de 30 cercarias de Schistosoma mansoni

\begin{tabular}{|c|c|c|c|c|c|}
\hline $\begin{array}{l}\text { Días después } \\
\text { la. infección }\end{array}$ & Grupo(*) & $\begin{array}{c}\text { No. de } \\
\text { hamsters }\end{array}$ & $\begin{array}{l}\text { Promedio de vermes inmaduros } \\
\text { recuperados } 14 \text { días después } \\
\text { de la roinfeccion }\end{array}$ & $\begin{array}{c}\% \\
\text { Protección }\end{array}$ & $\begin{array}{c}\text { Valores de } \\
P\left({ }^{* *}\right)\end{array}$ \\
\hline 40 & $\begin{array}{l}\mathbf{A} \\
\mathbf{B}\end{array}$ & $\begin{array}{l}12 \\
11\end{array}$ & $\begin{array}{l}43,2 \pm 10,3 \\
50,0 \pm 14,1\end{array}$ & 13,6 & N.S. \\
\hline 60 & $\begin{array}{l}\mathbf{A} \\
\mathbf{B}\end{array}$ & $\begin{array}{l}11 \\
12\end{array}$ & $\begin{array}{l}33,1 \pm 7,3 \\
51,4 \pm 14,3\end{array}$ & 35,6 & $<0,01$ \\
\hline 90 & $\begin{array}{l}\mathbf{A} \\
\mathbf{B}\end{array}$ & $\begin{array}{l}10 \\
11\end{array}$ & $\begin{array}{r}28,3 \pm 8,6 \\
48,9 \pm 14,0\end{array}$ & 42,2 & $<0,001$ \\
\hline 120 & $\begin{array}{l}\mathbf{A} \\
\mathbf{B}\end{array}$ & $\begin{array}{l}12 \\
12\end{array}$ & $\begin{array}{r}56,4 \pm 11,5 \\
101,0 \pm 26,0\end{array}$ & 44,2 & $<0,001$ \\
\hline
\end{tabular}

La reinfección a $\operatorname{los} 40,60$ y 90 días fue con cerca de 150 cercarias y a 105120 días con cerca de 250 cercarias. (*) A - Grupo experimental de hamsters que recibieron las dos infecciones. (*) B - Grupo control de hamsters que solo recibieron la segunda infección.

(**) Test $t$ de Student.

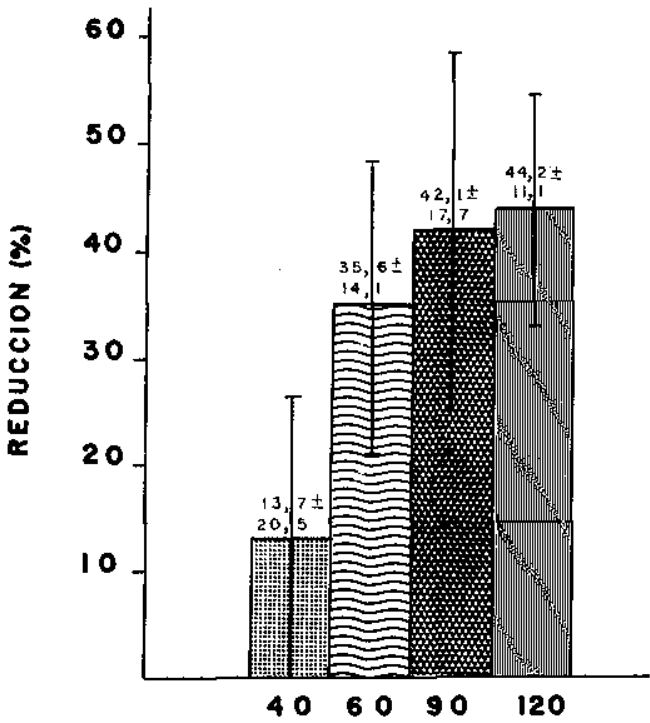

DIAS DESPUES DE LA INFECCION INICIAL

Fig. 1 - Reducción de la carga parasitaria de la relnfección de hamsters infectados inicialmente con cerca de 30 cercarias de Schistosoma mansoni. Cada punto representa el promedio de reducción de la carga parasitaria. Las líneas vertícales Indican la desviación estandar de los promedios.
La figura 2 nos muestra los porcentajes de esquistosómulos muertos por la acción citotó. xica del suero de los hamsters con diferentes periodos de infección. La letalidad fue estadísticamente menor con el suero de 40 días de infección cuando comparado con los sueros de 60 , 90 y 120 días $(P<0,001)$. Además hubo una diferencia significativa entre 60 y 90 días' $(P<$ $0,02)$ pero no entre los sueros de 90 y 120 días.

La concentración de anticuerpo letal estả relacionada con el tiempo de infección (Fig. 3). Así, en una dilución $1: 5$ el $33 \%$ de los esquistosómulos fueron lesionados con los sue ros de 90 y 120 días y apenas el $22 \%$ y el $16 \%$ con los sueros de 60 y 40 días respectivamente. Tal observación fue similar para los sueros di. luidos 1:10 y 1:20. Para ésta última, con suero de $\mathbf{4 0}$ días, la mortalidad fue negativa, indicando su baja concentración de anticuerpos; mientras que para los de 60,90 y 120 días era casi igual $(19-21 \%)$. El análisis de variancia solo fue sig. nificativo en la comparación del suero de 40 días de infección con el de 60, 90 y 120 días, er 
MONGE, E.; COELHO, P. M. Z. \& TAVARES, C. A. P. - Estudios inmunologicos en hamsters (Cricetus auratus) infectados con Schistosoma mansoni. Rev. Inst. Med. trop. São Paulo, 28:239-245, 1986.

las siguientes diluciones: $1: 5$ y $1: 10$ con sueros de 90 y $120(P<0,01)$ y de $1: 20$ con suero de 60 días $(P<0,05)$, de 90 días $(P<0,02)$ y con el de 120 días $(P<0,01)$.

Los hamster infectados y no infectados con S. mansoni fueron irmunizados y posteriormen. te probados inmunológicamente con glóbulos rojos de carnero. El Cuadro III resume los resultados obtenidos en estos experimentos indi. cando una diferencia significativa sólo a los 90 dias después de la infección inicial.

\section{DISCUSSION}

El hamster es un hospedero ideal para el S. mansoni tanto en el matenimiento del pará. sito como en el desarrollo de modelos experi. mentales. Por este motivo, lo estudiamos como posible modelo de laboratorio en la inmulologie de la esquistosomiasis mansoni, analizando du. rante 18 semanas diversos parámetros como la recuperación de vermes, la resistencia a la rein. fección, el efecto citotóxico del suero inmune y la hipersensibilidad retardada.

Diversos autores han señalado que el porcentaje de vermes recuperados en el hamster es alto en relación al número inicial de cerca. rias infectantes; nosotros logramos una recuperación de vermes adultos que alcanzó el $37,5 \%$ (Cuadro I) en concordancia con los re sultados señalados por FARIA \& PELLEGRI. NO ${ }^{5}$ y WARREN \& PETERS ${ }^{23}$.

La resistencia a la reinfección con $\mathbf{S}$. man. soni fue demonstrada por algunos autores 20 y desde entonces, son muchas las investigacio. nes en este sentido demostrando el desarrollo d inmunidad adquirida. A pesar de esto, no se han establecido los posibles mecanismos inmunológicos, humorales o celulares, por los cuales los animales se vuelven resistentes a la reinfección ${ }^{14}$. No obstante, los resultados sugieren que anticuerpos de la classe Ig G, los eosinófilos, los neutrófilos y los macrófagos están implicados en los mecanismos efectores de resistencia y paralelamente se ha señalado una acción sinérgica entre Ig $G$ y complemento? Sin embargo, NOVATO-SILVA \& col. 11 anotan que los esquistosómulos in vitro, ađquieren pro. tección contra el efecto letal de granulocitos y complemento, con o sin anticuerpo, aunque la preparación celular presente una alta concen tración de eosinófilos.

Similar a lo observado por SMITH \& col. ${ }^{18}$, en este estudio constatamos una reducción sig. nificativa de la carga parasitaria por desarrollo de resistencia a la reinfección (Fig. 2). SMITH \& CLEGG ${ }^{17}$, estudiando este problema en dos cepas de hamsters, informan que en una do ellas sólo se presentaron niveles de protección significativa en algunos de los 16 experimentos realizados, reduciéndose la carga parasitaria en aproximadamente $39 \%$. Por otro lado, cuando esta cepa fue reinfectada a los 42 días después de la primera infección no se encontró un nivel significativo de inmunidad. Nuestros hallaz. gos son similares pues los niveles de inmunida. dad variaron de 35,6 a $44,2 \%$ entre los 60 y 120 días y no se observó una protección signficativa a los 40 días después de la infección con 30 cercarias (Cuadro II).

Estos restultados también están de acuerdo con las observaciones de HUNTER \& col. ${ }^{6}$, que indican que varias dosis inmunizantes no son más efectivas que una sola con el mismo nú. mero de cercarias y edemás, que el tiempo transcurrido entre la infección primaria y la rein. fección es fundamental para que se desarrolle la resistencia. Igualmente, concuerdan con las de LONG \& col. ${ }^{7}$, en el sentido de que la in. munidad se adquiere de acuerdo con el desarrollo sexual de los vemes de la primera infección ya que es necesaria la ovoposición y además señalan que el grado máximo de resis. tencia depende de la intensidad de esa infec ción y que se manifiesta entre las primeras seis semanas o entre la 8." y la 19." semanas confor. me al mayor o menor número de cercarias utilizadas. En nuestro modelo se alcanzó después de la $6{ }^{a}$ semana de infección sugiriendo que la dosis de cercarias utilizadas en este trabajo es adecuada para los estudios propuestos.

Entre los numerosos estudios inmunológi. cos con S. mansoni, resaltan los de CLEG \& SMITHERS ${ }^{3}$ quienes demostraron, en el suero hiperinmune de monos Rhesus, la existencia de un anticuerpo letal, de la classe Ig G y dependiente del complemento, que actúa sobre los esquistosómulos. También fue hallado en conejos, ratas y ratones inmunizados con multi ples infecciones por cercarias y aún en huma. nos ${ }^{21}$. En nuestro trabajo demostramos por 
MONGE, E.; COELFO, P. M. Z. \& TAVARES, C. A. P. - Estudios inmunologicos en hamsters (Cricetus auratus) in fectados con Schistosoma mansoni. Rev. Inst. Med. trop. São Paulo, 28:239-245, 1986.

primera vez la presencia de este antícuerpo en el suero de hamsters infectados con el mismo parásito, el cual provoc6 una mortalidad de $38,2 \%$ y de $33,4 \%$ a los 90 y 120 días respecti. vamente, después de la infección inicial (Fig. 2); análogo a lo obtenido en ratones por TAVA.

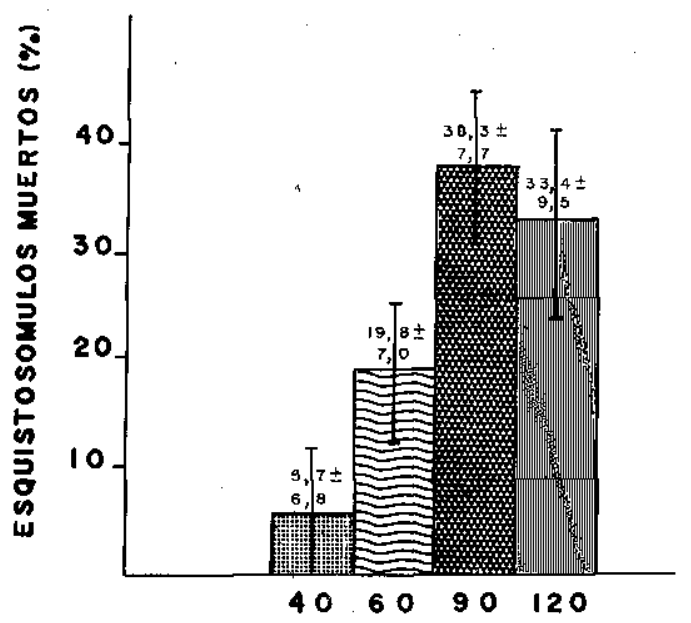

\section{DIAS DESPUES DE LA INFECCION IMICIAL}

Fig. 2 - Indice de mortalidad de esquistosómulos tratados con suero inmune de hamsters obtenidos en diferentes períodos después de la infección inicial con cerca de 30 cercarias de Schistosoma mansoni. El indice de mortalidad lue estimado por diferencia entre los esquistosómulos muertos por el suero inmune más complemento y los muertos sólo por la accíón del complemento. Cada punto representa el promedio del porcentaje de esquistosómulos muertos. Las lineas verticales indican Ia desviación estandar de los promedios

RES \& col. 22 . Además, la actividad đe los sueros; diluidos o no, aumentó con el tiempo de infección, tenđiendo a alcanzar un "plateau" alrededor del $90 .^{\circ}$ día (Fig. 3 ); lo cual fue similar a lo observado por PEREZ \& col. ${ }^{14}$.

Es necesario señalar que la aparición del anticuerpo letal, en el suero de hamsters, aparentemente va paralela al desarrollo de la inmunidad adquirida, lo que podría indicar una relación entre ambos fenómenos y, aunque se ha mencionado que los factores humorales de. sempeñan un papel muy importante en los mecanismos por los cuales el hospedero desarrolla resistencia a las reinfecciones ${ }^{16}$, no se ha podido correlacionar la presencia de anticuerpo letal con la inmunidad adquirida 10,14,16. Nosotros encontramos que a los 40 días de infección el nivel de anticuerpo letal fue muy bajo y la inmunidad adquirida aparentemente no se de-

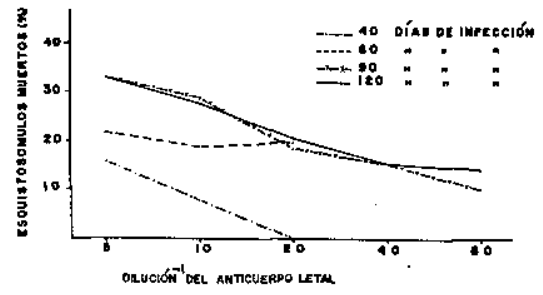

FIg. 3 - Títulos de anticuerpo letal en sueros de hamster obtenidos en diferentes perfodos después de lo infección inicial con cerca de 30 cercarias de Schistosoma manson. El indice de mortalidad fue estimado por diferencia entre Ios esquistosómulos muertos por el suero inmune mas complemento y los muertos solo por la ación del comple. mento. Cada punto representa el promedio del porcentajo de esquulstosómulos muertos en cada una de las diluciones

sarrolló, mientras que a $\operatorname{los} 60,90$ y 120 días si se demostró la presencia de tal inmunidad al aumentar significativamente el índice de mortalidad de los esquistosómulos. Podemos entonces inferir qu el hamster, como otros animales, es estimulado por el S. mansoni, producien. do un anticuerpo citotóxico para los esquistosomulos cultivados in vitro y cierto grado de resistencia a la reinfeccion. Sin embargo, a pesar de la estrecha semejanza entre el aumento de la inmunidad adquirida y los niveles de anticuerpo letal, no fue posible comprobarlo estađísticamente por medio de correlación linear.

Un aspecto de grande interés en la inmunologia de la esquistosomiasis es la evasión de la respuesta inmune por parte del parásito, por medio de varios mecanismos dentro de los que se podría incluir a la inmunosupresión. En el presente trabajo, durante la esquistosomiasis crónica inducida en hamsters, se observó una disminución de la hipersensibilidad retardada a la inoculación con eritrocitos de carnero, evidenciando una supresión de la funcionalidad de las células T auxiliares. MOTA-SANTOS \& col.9 demostraron, en ratones, que los vermes adultos o sus productos pero no sus huevos, causan una profunda alteración en el sistema inmune y que el fenómeno además, es dependiente de la carga parasitaria puesto que no se observa en infecciones leves antes de la ovoposición pero sí se manifiesta a los 85 dias después de la infección aún con una carga leve de parásitos Coincidiendo con esto, en el Cuadro III, se nota que la inmunosupresión sólo fue detectada a los 90 días después de la infección y no fue posible detectarla a los 60 días después de la primoinfección contrariamente a los resultados 
MONGE, E.; COELHO, P. M. Z. \& TAVARES, C. A. P. - Estudios inmunologícos en hamsters (Cricetus auratus) infectados con Schistosoma mansoni. Rev. Inst. Med. trop. São Paulo, 28:239-245, 1986.

C U A D RO III

Reaccion de hipersensibilidad celular en diferentes periodos después de la infeccion inicial de hamsters con cerca de 30 cercarias de Schistosoma mansoni

\begin{tabular}{cccc}
\hline $\begin{array}{c}\text { Dfas después } \\
\text { infección inicial }\end{array}$ & $\begin{array}{c}\text { "hinchazón de la pata"( } \\
\text { infectados }\end{array}$ & $\begin{array}{c}\text { Valores de } \\
\mathbf{P ( * * )}\end{array}$ \\
\hline 45 & $0,27 \pm 0,14$ & $0,38 \pm 0,10$ & N.S. \\
60 & $0,43 \pm 0,22$ & $0,64 \pm 0,34$ & N.S. \\
90 & $0,38 \pm 0,14$ & $0,86 \pm 0,27$ & $<0,05$ \\
\hline
\end{tabular}

(*) Promedio 't cesviación estándar de 5 hamsters

(**) Test $t$ de Studient

obtenidos por otros autores 1,4 , probablemente debido a un fenómeno dependiente de la car. ga parasitaria.

Los resultados obtenidos, a la par del he cho de ser el hamster un animal ampliamente usado en investigación científica debido a su pequeño porte y su fácil manejo; indican a este animal como un modelo alternativo interesante para estudios inmunológicos sobre esquistosomiasis.

\section{SUMMARY}

Immunologic studies in hamsters (Cricetus au. ratus) infected with Schistosoma mansoni

The results of this investigation show that the hamsters (Cricetus auratus) may be used as an experimental model for immunological studies in Schistosoma mansoni. The data ob. tained referring to concomitant immunity, procuction of lethal antibodies and immunosup. pression, resemble data from other established experimental models.

Our observations indicate that the hams. ter has the following advantages: 1 . It is an appropriate host for the maintenance of $\mathbf{S}$. mansoni in the laboratory. 2. It can be consi. dered an alternative model for easy growth and maintenance of this parasite. 3. It is easy to handle.

\section{REFERENCIAS}

1. ARAUJO, F. G.; COELhO, P. M. Z.; PEREIRA, L. H. \& PELLEGRINO, J. - Schistosoma mansoni: impair. ment of the cell-mediated immune response in mice. Clin. exp. ImununoI., 28: 289-291, 1977.

2. BUTTERWORTH, A. E. - Effector mechanisms against schistosomes in vitro. Amer. J. trop. Med. Hyg., 26 (Suppl.): 29-35, 1977.
3. CLEGG, J. A. \& SMITHERS, S. R. - The effect of immune rhesus monkeys serum on schistosomules of Schistosoma mansoni during cultivation in vitro. Int. J. Parasit., 2: 79-98, 1972.

4. COELhO, P. M. Z.; MAYRING, W.; DIAS, M. \& PEREIRA, L. H. - Susceptibility to Leishmanla mexicana of mice infected with Schistosoma mansonl. Trans. roy Soc. trop. Med. Hyg., 74: 141, 1980.

5. FARIA, J. \& PELLEGRINO, J. - Observaçסes sobre a infecção experimental do hamster (Cricetus auratus) pelo Schistosoma mansoni. Rev. Inst. Med. trop. S. Paulo, 5: 281-286, 1963.

6. HUNTER III, G. W.; GRANDALL, R. B.; ZICKAFOOSE, D. E. \& PURVIS, Q. B. - Studies on schistosomiasis. XVIII. Some factors affecting resistance to Schistosoma mansoni infections in albino mice. Amer. J. trop. Med. Hyg., 11: 17-24, 1962.

7. LONG, E.; DOENHOFF, M. \& BAIN, J. - Factors affecting the acquisition of resistance against Schisto. soma mansonf in the mouse. 2. The time at which resistance to reinfections develops. J, Helminth., 52: 187-191, 1978.

8. MILLER, T. E.; MAGKANESS, G. H. \& IAGRANGE, P. H. - Immunopotentiation with BCG. II. Modulation of response to sheep red blood cells. J. nat. Cancer Inst., 51: 1669, 1973.

9. MOTA-SANTOS, T. A.; TAVARES, C. A. P.; GAZZINELLI, G. \& PELLEGRINO, J. - Immunosuppression mediated by adult worms in chronic schistosomiasis mansoni. Amer. J. trop. Med. Hyg., 26: 727-731, 1977.

10. MURREL, K. D.; DEAN, D. A. \& STAFFORD, E. E. - Resistance to infection with Schistosoma mansoni after immunization with worm extracts or live cercariae: Role of cytotoxic antibody in mice and guinea pigs. Amer. J. trop. Med. Hyg., 24: 955-962, 1975.

11. NOVATO-SILVA, E.; NOGUEIRA-MACHADO, J. A. \& GAZZINELLI, G. - Schistosoma mansoni: Comparison of the killing effect on fresh and cultured schistosomula in vitro. Amer. J. trop. Med. Hyg., 29: 1263-1267, 1980.

12. PEARCE ,E. J. \& MCLAREN, D. J. - Reappraisal of the guinea-pig as an experimental host for studies of schistosomiasis mansoni. Parasitology, 87: 456-464, 1983.

13. PEARCE, E. J. MCLAREN, D. J. - Schistosoma mansoni: in vivo and in vitro studies of immunity using the guinea-pig model. Parasitology, 87: 465-479, 1983.

14. PEREZ, H.; CLEGG, J. A. \& SMTTHERS, S. R. Acquired immunity to Schistosoma mansont in rat: Measurement of immunity by lung recovery technique. Parasltology, 69: 349-359, 1974.

15. RAMALHO.PINTO, F. J.; GAZZINELLI, G.; HOWELIS, S. R.; MOTA-SANTOS, T. A.; FIGUEIREDO, E. A. \& PELLEGRINO, J. - Schistosoma mansoni: Defined system for step-wise transformation of cercariae to schistosomule in vitro. Exp. Parasit., 36: 360-372, 1974

16. SHER, A.; SMITHERS, S. R. \& MACKENZIE, P. Passive transfer of acquired resistance to Schistosoma mansoni in laboratory mice. Parasitology, 70: 347-357, 1975. 
MONGE, E.; COELFO, P. M. Z. \& TAVARES, C. A. P. - Estudios inmunologicos en hamsters (Cricetus auratus) in. Pectados con Schistogoma mansoni. Rev. Inst. Med. trop. São Paulo, 28:239-245, 1986.

17. SMITH, M. A. CLEGG, J. A. - Different levels of acquired Immunity to Schistosoma mansoni in two strains of hamsters. Parasitology, 73: 47-52, 1976.

18. SMITH, M. A.; CLEGG, J. A.; KUSEL, J. R. \& WEBBE, G. - Lung inflammation in immunity to Schistosoma mansoni. Experimentia (Basel), 31: 595$596,1975$.

19. SMITHERS, S. R. \& TERRY, R. J. - Acquired resistaice to experimental infections of Schistosoma mansond In the albino rat. Parasitology, 55: 711-717, 1965,

20. SMITHERS, S. R. \& TERRY, R. J. - The immunology of schistasomiasis. Adv. Parasit., 7: 41-93, 1969.

21. SMTTHERS, S. R. \& TERRY, R. J. - The immunoloģ of schị̧țoșomiagsis, Adv. Parasit., 14: 339-422, 1976.
22. TAVARES, C. A. P.; GAZZINELII, G.; MOTA-SANTOS, T. A. \& DIAS DA SILVA, W. - Schistosoma mansoni: Complement-mediated cytotoxic activity in vitro and effect of decomplementation on acquired immunity in mice. Exp. Parasit., 46: 145-151, 1978.

23. WARREN, K. S. \& PETERS, P. A. - Comparison of penetration and maturation of Schistosoma mansonl in the hamster, mouse, guinea-pig, rabbit and rat. Amer. J. trop. Med. Hyg., 16: 718-722, 1967.

24. WHO-MEMORANDUM - Immunology of schistosomia. sis. Bull. Wld. HIth. Org., 51: 553-595, 1974.

Recebtdo para publicação em 27/8/1985. 